with a detectable relapse clone at diagnosis $(P=0.004)$. Kaplan-Meier survival analysis confirmed that the presence of relapse clones at diagnosis significantly correlated with relapse-free survival $(P<0.007)$. In cases in which quantification of the clones was possible, high levels of the relapse clone at the time of diagnosis correlated with a shorter remission $(r=-0.84 ; P=0.018)$.

The findings of this study suggest that relapse might involve the expansion of a minor tumorigenic cell population that is present at diagnosis. Early identification of these resistant subclones during treatment might result in improved relapse-free survival in children with ALL.

Original article Choi S et al. (2007) Relapse in children with acute lymphoblastic leukemia involving selection of a preexisting drug-resistant subclone. Blood 110: 632-639

\section{Association between radiation- induced xerostomia and QOL in head and neck cancer}

Xerostomia is the most frequently reported late side effect of radiation for the treatment of head and neck cancer. Uncertainty regarding the clinical relevance of radiation-induced xerostomia in relation to patient quality of life (QOL) led Jellema et al. to test the following two hypotheses: firstly, that higher grades of radiation-induced toxicity correlate with worse QOL; and secondly, that the impact of radiation-induced xerostomia on overall $\mathrm{QOL}$ lessens with time.

Late xerostomia (assessed with the RTOG Late Radiation Morbidity Scoring System) and QOL (EORTC QLC-C30 scale) were evaluated at baseline and every 6 months from 6 to 24 months after radiotherapy in 288 patients with stage I-IVB head and neck cancer without distant metastases. At 6 weeks, approximately two-thirds of patients had xerostomia. The presence of xerostomia was significantly associated with several QOL measures, including overall QOL outcome, physical functioning, social functioning, fatigue and insomnia. The magnitude of the effect seemed to be dependent on age and sex-women were more affected than men for several QOL measures (possibly because women are more prone to insomnia, which could exacerbate some of the effects), while younger patients were more affected in physical and social functioning than were older patients with the same grade of xerostomia. Although the incidence of xerostomia decreased with time, its effect on overall QOL increased (effect size 0.09 at 6 months, rising to 0.22 at 24 months)

The prevention of radiation-induced xerostomia could, therefore, result in marked improvements in QOL for these patients.

Original article Jellema AP et al. (2007) Impact of radiation-induced xerostomia on quality of life after primary radiotherapy among patients with head and neck cancer. Int J Radiat Oncol Biol Phys [doi: 10.1016/j.ijrobp.2007.04.021]

\section{Study identifies risk factors for progression of smoldering multiple myeloma}

Patients with smoldering multiple myelomaan asymptomatic proliferative disorder of plasma cells - are at high risk of progression to active multiple myeloma. In a recent paper, Kyle et al. report that the risk of progression to symptomatic disease is associated with the proportion of plasma cells in the bone marrow, and the serum monoclonal protein level, at diagnosis.

The researchers searched a computerized database and Mayo Clinic medical records and identified 276 patients who fulfilled the criteria of the International Myeloma Working Group for the diagnosis of smoldering multiple myeloma. The median age at diagnosis was 64 years, with $62 \%$ of the cohort being male. Over a cumulative follow-up of 2,131 personyears, $57 \%$ of the population progressed to active multiple myeloma and $2 \%$ developed amyloidosis. Median survival following a diagnosis of active myeloma was 3.4 years. The overall risk of progression to symptomatic disease was $10 \%$ per year for the first 5 years, $3 \%$ per year for the next 5 years, and $1 \%$ per year for the next 10 years, resulting in a cumulative probability of progression to active myeloma of $73 \%$ at 15 years. Multivariate analysis identified serum monoclonal protein level and proportion of plasma cells in the bone marrow as significant independent risk factors for disease progression; hemoglobin, sex, serum albumin level, proportion of normal 